Western University

Scholarship@Western

Aboriginal Policy Research Consortium International (APRCi)

2010

\title{
A Four-Stage Method for Developing Early Interventions for Alcohol Among Aboriginal Adolescents
}

Christopher J. Mushquash

Brian D. McLeod

Sherry H. Stewart

Follow this and additional works at: https://ir.lib.uwo.ca/aprci

Part of the Substance Abuse and Addiction Commons

Citation of this paper:

Mushquash, Christopher J.; McLeod, Brian D.; and Stewart, Sherry H., "A Four-Stage Method for Developing Early Interventions for Alcohol Among Aboriginal Adolescents" (2010). Aboriginal Policy Research Consortium International (APRCi). 257.

https://ir.lib.uwo.ca/aprci/257 


\title{
A Four-Stage Method for Developing Early Interventions for Alcohol Among Aboriginal Adolescents
}

\author{
Christopher J. Mushquash • M. Nancy Comeau • \\ Brian D. McLeod • Sherry H. Stewart
}

Received: 27 May 2009 / Accepted: 5 August 2009 /

Published online: 18 August 2009

(C) Springer Science + Business Media, LLC 2009

\begin{abstract}
This paper details a four-stage methodology for developing early alcohol interventions for at-risk Aboriginal youth. Stage 1 was an integrative approach to Aboriginal education that upholds Aboriginal traditional wisdom supporting respectful relationships to the Creator, to the land and to each other. Stage 2 used quantitative methods to investigate associations between personality risk factors and risky drinking motives. Stage 3 used qualitative interviews to further understand the contexts and circumstances surrounding drinking behaviour within a larger cultural context. Stage 3 involved tailoring personalitymatched, motive-specific brief interventions to meet at-risk adolescents' needs. Stage 4 involved an efficacy test of the interventions. This novel methodology has significance for future program development to meet diverse social, cultural and health needs of at-risk adolescents.
\end{abstract}

Keywords Drinking motives · Aboriginal adolescents · Early intervention ·

Personality risk $\cdot$ Alcohol abuse

Aboriginal youth can experience a "spirit of belonging" with their identity and culture (Ball 2006; Brokenleg 2002; Aboriginal Healing Foundation 2004, 2005, 2006).

In a report by the Aboriginal Healing Foundation (2004), it was stated that, "In order for Aboriginal people to devise culturally appropriate healing modalities that will help them to overcome social disorders resulting from the historic trauma they experienced, a people centered and a people directed approach has to be adopted" (Aboriginal Healing Foundation 2004, p. 77). The "Voices of the Elders" speak to this spirit of belonging:

Aboriginal educators and Elders have envisioned an education for their children that strengthens and inspires by focusing on traditional wisdom. They have envisioned an education where the young people of today are helped in creating a peaceful balance

C. J. Mushquash $(\bowtie) \cdot$ M. N. Comeau $\cdot$ S. H. Stewart

Dalhousie University, Halifax, NS, Canada

e-mail: chris.mushquash@dal.ca

B. D. McLeod

Strong Heart Teaching Lodge, Winnipeg, MB, Canada 
within themselves using Aboriginal "laws" as a guide. The "laws" which govern life, are not laws in the literal and mechanistic sense. They are perspectives that can help young people to orient themselves positively as Aboriginal people while establishing or strengthening their personal identities. They are perspectives that enable Aboriginal people to live with integrity, regardless of the environment or circumstances in which they find themselves. (Ministries of Education Working Group 2000, p. 10)

Broadly, people-centered and people-directed approaches relate to a grassroots philosophy wherein individuals and communities are determinative, and, around which all else is structured when it comes to the development, implementation, and evaluation of supporting systems of services. A people-focused wisdom of the community involves treating others as related, a social value that has transformative power for human relationships (Brokenleg 2002). Following Brokenleg (2002), the four-stage methodology featured in this paper highlights the importance of relationships wherein this sense of belonging extends to nature as well. Within the scope of this methodology, the communitydriven process of relationship building with researchers is primary.

Research agendas that encompass comprehensive interventions are needed to prevent and intervene early with alcohol misuse among adolescents in racially, ethnically, and economically diverse urban and rural communities (Abrams and Clayton 2001). Various epidemiological studies have documented high levels of mental health and substance abuse problems in many Canadian First Nations communities (Kirmayer et al. 2001). Among the social problems indicated by First Nations people as a concern in their community is substance abuse. Moreover, alcohol and other drugs have been identified as leading causes of adolescent morbidity and mortality consequent to motor vehicle accidents, suicidal behaviour, violence, falls, drowning, and unprotected sex (Chandler et al. 2003; Kaminer 1999). As such, youth alcohol and other substance abuse in First Nations communities is an important community and public health concern and can be readily understood in part as the direct consequences of broad social factors, i.e., dislocations and disruption of traditional subsistence patterns and connection to the land (Kirmayer et al. 2001) as well as individual level variables.

Motivational theories of substance abuse variability generally propose that individual differences in personality reflect different susceptibilities to certain reinforcing properties of drugs of abuse (Cooper et al. 1995; Conrod et al. 2000a; Pihl and Peterson 1995). Conrod et al. (2000a) found support for the validity of this motivational hypothesis by demonstrating that substance-abusing women, classified according to specific personality profiles (e.g., anxiety sensitivity [AS: fear of anxiety-related symptoms], hopelessness/negative thinking [HOP: tendency toward feelings of despair and/or worthlessness] and sensation seeking [SS: desire for intense and novel experiences]), manifested distinct patterns of addictive and non-addictive psychopathology and coping skills deficits (e.g., avoidance behaviour and problematic alcohol use).

High AS individuals may attempt to deal with stressful physical sensations and their worry about anxious feelings by avoiding situations where anxiety may occur. Another style of maladaptive coping to which high AS individuals are susceptible is regular heavy drinking to feel calm and less anxious. High HOP individuals tend to drink to "numb" negative feelings. In contrast, a high SS individual who craves excitement and often acts without thinking clearly about the consequences may avoid boredom by drinking heavily as a way of responding to the urge to do something exciting (Comeau 2004; Conrod et al. 2000b; Mushquash et al. 2007; Theakston et al. 2004; Stewart and Devine 2000; Stewart et al. 2001). Findings from studies examining adults from the majority culture with substance 
use disorders (e.g., Conrod et al. 2000a) have highlighted the potential importance of developing intervention strategies that differentially target subtype-specific motivational, personality, and coping skills profiles (Conrod et al. 2000b). Brief interventions involving cognitive behavioural coping skills training are most effective in treating substance abuse disorders when they are applied in a "matched" fashion (Conrod et al. 2000b). For example, SS substance abusers benefit most from coping skills that specifically target their underlying enhancement drinking motives: drinking for reasons of increasing internal positive emotional states. Comeau and colleagues extended this treatment approach for use in school-based early intervention with at-risk groups of AS, HOP, and SS teenage drinkers (Comeau 2004; Comeau et al. 2004; Conrod et al. 2006). A similar rationale informed the development of this four-stage methodology for First Nations teens at high personality risk of alcohol misuse.

Quantitative research provides an empirical case for targeting personality factors as a means for reducing "risky" drinking motives in adolescents (Cooper 1994). Coping, conformity, and enhancement motives are considered "risky" due to their established associations with heavy drinking and/or drinking related problems in adolescents (Comeau et al. 2001, 2004; Conrod et al. 2006; Cooper 1994) and young adults (Conrod et al. 1998, 2000b; Stewart et al. 1997, 2001; Stewart and Zeitlin 1995). These maladaptive motives in turn put a young person at risk for alcohol problems (i.e., specific types of harm resulting from alcohol use). AS, HOP, and SS are each associated with a different maladaptive drinking motive (i.e., coping/conformity, coping, and enhancement, respectively; Cooper 1994; Comeau et al. 2001; Comeau 2004; Stewart and Zeitlin 1995). Thus, some have suggested that by intervening at the level of personality vulnerability, one can change or help manage at-risk teens' maladaptive drinking motives (e.g., coping, conformity, and enhancement; Cooper 1994) and ultimately reduce heavy drinking and alcohol related problems (Pihl and Peterson 1995; cf. Comeau 2004). Organizationally, this approach selects first for personality factors and then targets maladaptive coping including problematic drinking motives (Conrod et al. 2006).

The methodology presented in this paper uses the apparatus of quantitative and qualitative research to pursue understanding of drinking patterns, contexts, and consequences particular to First Nations adolescents at high personality risk of alcohol abuse. While large-scale quantitative surveys are helpful for determining the incidence of drinking and comparing behaviour patterns of teens, qualitative research approaches are also needed to capture important variations across adolescents' diverse social interactions. This methodology aims to take into account the contexts in which adolescents live and develop (i.e., broad social contexts in which alcohol campaigns are frequently aimed at youth) as well as self-perceived reasons for drinking as expressed in teens' own words. This paper will detail how we have combined results from quantitative and qualitative investigations to develop a unique set of interventions for preventing alcohol misuse in at-risk First Nations teenage drinkers which are meaningful to the lives of these adolescents (see also Comeau 2004; Comeau et al. 2004). Together, the studies reported in this paper were designed to add insight into our understanding of high risk adolescents' relationships with alcohol and explore four inter-related questions: 1) How does personality relate to reasons for drinking for Aboriginal adolescents?; 2) How do these youth, at particular risk of alcohol abuse, understand their relationships with alcohol?; 3) Can established alcohol abuse brief early interventions be effectively tailored to meet the needs of high personality risk Aboriginal teens?, and 4) Do interventions developed specifically for Aboriginal youth with varying personality risk characteristics and maladaptive motives for alcohol use effectively reduce drinking behaviour and problems associated with alcohol use? 
There are four stages to this approach: Stage 1-integrative Aboriginal education and knowledge exchange; Stage 2-quantitative (study 1); Stage 3-qualitative (study 2) and manual development for brief interventions, and; Stage 4-outcome evaluation (Mushquash et al. 2008). We applied this unique 4-Stage methodology to develop a culturally relevant early intervention program for adolescent First Nation drinkers high in the personality risk factors of AS, HOP, and SS (Comeau et al. 2005c). This research with Mi'kmaq youth was based on a methodology that was previously used to inform the development (Comeau 2004; Comeau et al. 2004) and testing (Comeau 2004; Conrod et al. 2006) of intervention techniques for at-risk youth more generally.

\section{Stage 1}

Stage 1 encompassed a consultation process with individuals from the community who had a strong knowledge base of the community and its youth support services. The Royal Canadian Mounted Police (RCMP) Aboriginal and Diversity Policing Services " $\mathrm{H}$ " Division (Nova Scotia), Community Policing Services "H" Division, and First Nations school-based partners, indicated a need for culturally relevant programming that addresses problems of adolescent substance abuse. In 2002, two local committees of schoolcommunity partners met at two respective Mi'kmaq First Nation schools to share concerns and potential strategies for community collaboration toward prevention and intervention of problems related to alcohol and other drug use.

An exciting pilot project resulted entitled, "Nemi'simk, Seeing Oneself", which included a novel set of student and facilitator manuals that seeks to prevent alcohol and other substance misuse by at-risk Aboriginal teens-via brief school based interventions that are deeply meaningful to the lives of these adolescents (Comeau et al. $2005 \mathrm{a}, \mathrm{b}$ ). The project facilitates program delivery via trained community members as facilitators and co-facilitators. First Nations school guidance counsellors received training as program facilitators. RCMP members of the Aboriginal and Diversity Policing Services were also trained as co-facilitators to accompany school guidance counsellors in a supportive non-clinical role.

The methodology on which the pilot project (Comeau 2004; Comeau and Stewart 2005) is based has inter-provincial and national significance for future program development to meet diverse cultural and health related needs of adolescents in Aboriginal communities in affiliation with the RCMP and other policing partners. In line with the Government of Canada's priority to build stronger Aboriginal communities, the RCMP, provincial police forces and First Nations police forces are committed to bring a greater focus to this area of policing by dealing with the various challenges Aboriginal peoples face both on and off reserves. This collaboration is timely considering the disproportionately high level of tragic suffering from alcohol and other drug abuse and suicide among Aboriginal peoples across Canada (Chandler et al. 2003). Further, this collaboration aims to provide mechanisms for creative methods of promoting crime and substance abuse prevention and improved mental health, particularly among Aboriginal young people. Thus, strong links to partners in the community, including schools and other social or youth oriented agencies, is a priority. The Royal Canadian Mounted Police Foundation (MPF) was a primary sponsor of this pilot project. The generous MPF contribution was matched by funding support from Dr. Cheryl Bartlett, the Canada Research Chair in Integrative Science at Cape Breton University (CBU). Integrative Science was proposed in the mid 1990s by individuals in the science community at $\mathrm{CBU}$ and at Eskasoni (the largest First Nations Community east of 
Montreal). This four step methodology is inspired by the objectives of the Canada Research Chair in Integrative Science specifically related to this pilot project as follows:

1. to extend capacity to bring together "ways of knowing and knowledges" of Aboriginal peoples and Western science;

2. to ensure scientific and cultural integrity by explicating modes and patterns of thinking, thereby enabling respectful acknowledgement of different cognitive tools;

3. to ensure scientific and cultural integrity by consulting and collaborating with others;

4. to disseminate new knowledge broadly in Aboriginal communities and in society at large.

The community consultation process spanned the methodology components. It was essential that respectful relationships be encouraged and maintained with individuals in the community seen as carriers of traditional knowledge (i.e., cultural resource people, Elders, traditional advisors). When researchers talked with Elders it was important that the researchers uphold cultural protocols (e.g., listen without interruption when an Elder spoke). Special attention was paid to the integration of traditional knowledge and its effective use with respect to contemporary issues facing Aboriginal youth. The ability of the youth to make these connections to every aspect of their life is essential to the increased likelihood of their success as productive members of their community and society as a whole. A process of integrative Aboriginal education was advanced which supported a collective world view that connects to the land and all living things, honouring relatives and relations in keeping with the thought that we all work as equals to one another. Respect for the relevancy of traditional teachings provided seeds of integrity from which youth can grow and develop themselves.

\section{Stage 2}

\section{Method}

Stage 2 involved school based questionnaire administration within two Mi'kmaq communities in Nova Scotia to determine the extent to which previously demonstrated personality-motives relations (Comeau et al. 2001; Comeau 2004; Conrod et al. 2006) are valid for Mi'kmaq youth. Study information was distributed to parents/guardians of students in grades 8-12 in participating schools. Parental authorization was provided through a "negative consent" procedure (i.e., parents were asked to contact the researcher or the school guidance counsellor for any further information about the study and were asked to let the researcher/guidance counsellor know if they did not consent to having their child participate). Parents/guardians were provided with a toll free number to contact the researcher. There were no parents/ guardians who declined consent for their children to participate. Prior to testing, students were informed about the nature of the study, and willing students provided written informed consent at the time of data collection. Across all data collection sites, the general questionnaire completion process was conducted in an identical fashion. Specifically, student consent forms indicated that the student's participation was voluntary, and that the youth's confidentiality would be maintained. Data collection was conducted on a grade-by-grade basis during class time. During questionnaire completion, students were permitted to ask questions of the researchers. 


\section{Measures}

The Substance Use Risk Profile Scale (SURPS) assesses levels of four personality risk factors for substance abuse/dependence (i.e. anxiety sensitivity. hopelessness, sensationseeking, impulsivity). The SURPS contains non-overlapping items between the risk factors and has been shown to have good convergent, discriminant, and factorial validity in adults and adolescents from majority culture (Conrod et al. 2000a, b).

The Drinking Motives Questionnaire-Revised (DMQ-R; Cooper 1994) is a 20-item selfreport measure designed to tap levels of the four drinking motives in Cooper's model of drinking motivations (i.e. coping, conformity, enhancement, and social). It has good psychometric properties in majority culture and First Nations adolescent samples (Cooper 1994; Mushquash et al. 2008).

\section{Results}

A total of 164 First Nation adolescents from two Mi'kmaq communities ( 85 girls; 79 boys), ages 14-18 years $($ mean age $=16.3)$, from grades 8 through $12($ mean grade $=10.1)$ completed the questionnaires. Of the 164 participants, $96(58.5 \%)$ reported using alcohol in last 4 months which was the eligibility criteria for inclusion in the analyses. Preliminary investigation of associations between personality factors and drinking motives using Cooper's (1994) categories of substance use motivations as applied to Mi'kmaq teens' use of alcohol indicated a distinction between two particular pathways of personality risk among First Nation youth. These risk pathways involve increased likelihood that a young person will consume alcohol for specific maladaptive drinking motives, namely enhancement, coping, and conformity (c.f. Cooper 1994). Overall, findings supported differential pathways of SS to enhancement motives, HOP to coping motives, and AS to the negative reinforcement motives of coping and conformity (Mushquash et al. 2008; Comeau et al. 2005a, b, c).

\section{Stage 3}

\section{Method}

Stage 3 involved conducting qualitative interviews with Mi'kmaq youth at high personality risk of substance abuse. These qualitative interviews permitted teens to speak in their own words without the constraints of pre-determined drinking motives categories and allowed for the identification of themes related to drinking motives of AS, HOP, and SS First Nations teenage drinkers. This stage further enlisted the engagement of teenage drinkers at high personality risk for alcohol abuse (i.e., AS, HOP, or $\mathrm{SS}^{1}$ ). Stage 3 focused on exploring personality — drinking motives relationships from teens' own perspectives. Using the results of the questionnaires administered in Stage 2, teens who were drinkers and who scored high on AS, HOP or SS were invited to participate in individual qualitative interviews. A "drinker" was defined as an individual who reported having consumed alcohol at least once in the past 4 months prior to the screening survey. On average,

\footnotetext{
1 "Impulsivity" is an additional personality-risk for alcohol and other substance abuse (Conrod et al. 2000a, b). For further discussion see Castellanos and Conrod 2006. This personality risk factor is yet to be investigated in relation to First Nations adolescents' alcohol use and misuse.
} 
participants in the AS, HOP and SS groups were drinking five or more drinks at each drinking occasion approximately once per week - hence a frequent heavy drinking sample (i.e., five drinks per occasion is usually considered heavy drinking; e.g., Poulin and Wilbur 2002).

Qualitative interviews in Stage 3 explored in greater depth, findings from Stage 2 as well as whether the expected associations between HOP and coping motives would emerge in qualitative interviews. A one-on-one qualitative interview was employed to hear from these three groups of high personality risk adolescents how they conceptualize alcohol use in a social context - in particular, how these teens' experiences of personality, motives, and other social factors influence their understandings of why they drink.

A representative group of adolescents scoring high in these personality factors were recruited from the overall Stage 2 group $(n=164)$ to participate in a face-to-face qualitative interviews investigating Mi'kmaq First Nation teens' motives for alcohol use, alcohol use contexts, and perceived relations of personality to drinking behaviour. The qualitative component (Comeau and Stewart 2004) was based on the assumption that open-ended, semi-structured interviews would yield results consistent with, but substantially extending, previous questionnaire-based research (Comeau and Stewart 2004; Comeau et al. 2005a, b, c; Stewart et al. 2005a, b, c, d) on the motives underlying alcohol use behavior in AS, SS, \& H Mi'kmaq First Nation adolescents. Eight at-risk First Nation adolescent drinkers (3 AS, $3 \mathrm{H}$ and $2 \mathrm{SS} ; 4$ boys, 4 girls) participated in one to one interviews which included open-ended questions. Interviews followed a semi-structured format using a portion of the Comprehensive Drinker Profile that provides seven open-ended questions related to drinking motives or reasons for drinking (CDP; Marlatt and Miller 1984). The following two questions were added to gather further information that would not necessarily be elicited in a CDP-only interview ${ }^{2}$ :

i./ Your personality can be defined as qualities and characteristics that make you who you are and make you different from others. I would like you to think for a moment about yourself and your personality. I would like you to describe for me the qualities and characteristics that make you who you are and make you different from others.

ii./ Do you think your personality has anything to do with your reasons for drinking alcohol?

Although these open-ended questions guided the interview, participants were able to go in whatever direction and pursue any ideas they chose as they told their stories. The interviews were conducted during school time. Responses during the sixty minute audiotaped interviews were transcribed verbatim and coded for theoretically relevant themes and novel narratives using qualitative research software (Ethnograph; Scolari 1999).

Results

Results of interviews were consistent with and substantially extended our Stage 2 (Study 1) questionnaire-based (quantitative) study findings (Comeau et al. 2005a, b, c). Personality-group specific themes emerged. Drinking motives were investigated by examining AS, HOP, and SS teens' internal (personal) and external (social context)

\footnotetext{
${ }^{2}$ The first 7 open-ended questions were from 'Motivational Information-Reasons for Drinking' of the "Comprehensive Drinker Profile"; Marlatt and Miller (1984). The Comprehensive Drinker Profile. Odessa, FL: Psychological Assessment Resources, Inc. The last 2 questions were author-generated (Comeau 2004).
} 
reasons for alcohol use (Cooper 1994). Internal reasons included drinking to obtain a desired outcome that involves mood alterations (i.e., decreasing negative mood states or increasing positive mood states). External reasons included drinking to achieve an external reward or obtain a positive outcome (e.g., social approval) or to avoid a negative outcome (e.g., being labelled as "not cool" or being left out of a group by friends who are drinking). For example, social context factors helped clarify whether an SS individual was drinking because of boredom or restlessness or due to craving more excitement in a highly stimulating social situation.

The personality constructs of AS, HOP and SS and their relationship to drinking motives were both supported and broadened in the qualitative data. For example, results from the AS interviews included an association between negative reinforcement motive of conformity (Cooper 1994). AS participants talked about drinking to lessen worries about feeling anxious and reported appreciating alcohol because drinking helped them feel less anxiety about fitting in with others in social situations. In addition, there was an association between coping motivated drinking (Cooper 1994) and HOP, with youth reported that drinking reduced feelings of sadness about other immediate concerns. There was a strong theme of negative affect reduction in the HOP youth. Participants in the HOP group particularly appreciated that alcohol had the effect of numbing bad feelings. Among the SS youth there was an association between enhancement motivated drinking (Cooper 1994) and SS. A predominant theme of positive affect enhancement was evident in terms of SS teens' specific motives for drinking. This group of teens spoke about drinking to enhance feelings of fun and having a good time. Drinking enhanced their enjoyment of a situation by maximizing the pleasure in the moment. One SS youth stated, "It's pretty cheap and I like laughing...you get the laughs...that's a good thing about it." (SS) Another SS youth stated, "You can loosen up and have fun."

Similarities across personality groups highlighted First Nations teens' strength, agency, and resistance to sexual exploitation, racial discrimination, and threat of violence. Such stresses as produced by exposure to violence and associated powerlessness appear associated with some teens' reasons for drinking. Teens who witnessed violence also spoke of a strong dislike or resistance to heavy alcohol use by others. Among peers, incidence of physical violence occurred when friends were drinking and fights broke out. Use of "pills" and marijuana was frequently cited in such situations.

Student and facilitator manuals were developed in Stage 3 to include cognitive behavioural techniques modified from previously tested manuals for adolescents and adults from the majority culture (Comeau 2004; Conrod et al. 2000b, 2006). However, the content was modified based on input from the qualitative semi-structured interviews with groups of AS, HOP and SS teenage drinkers as described above, as well as understanding gleaned from the quantitative data collected in Stage 2. The manuals developed in Stage 3 of this methodology were therapeutic in nature and the associated intervention program can be classified as a 'targeted' with the goal of reducing alcohol involvement among at-risk youth and stemming the progression of alcohol misuse (Comeau 2004; Comeau et al. 2004; Kaminer 1999). This approach has been shown to delay the escalation of drinking and binge drinking over time when applied with youth from the majority culture (Conrod et al. 2008). Illustrative stories and images in the Aboriginal manuals were informed by quantitative findings from Stage 2 and the results of the qualitative interviews in Stage 3. Creative images from Mi'kmaq teen artists illustrating the stories enriched manual content (Comeau et al. 2005a, b, c).

The set of interventions used in this "Seeing Oneself" initiative incorporated the integration of both Aboriginal content and perception within an approach to skills 
training which aims to equip different groups of high risk young people with coping skills to negotiate between mainstream and Aboriginal cultures. Aboriginal culture was not simply an "add on" to program content but was interwoven throughout intervention programming to entail a deeper understanding of cultural values, practises (which describe a way of life), and symbols. The intervention programming combined culturally tailored content and activities with cognitive-behavioural skills development. The four main components of our set of interventions were (a) culturally grounded content, (b) psycho-education, (c) behavioural coping skills training, and (d) cognitive coping skills training (cf. Comeau 2004; Conrod et al. 2006), all of which were delivered with culturally-tailored content and methods. Culturally grounded content and activities throughout the manual helped to describe the Aboriginal world-view and helped youth to integrate Aboriginal perspectives and content into all areas of their life. In an interactive manner, the psycho-education component involved youth being educated about links between the personality factor in question and alcohol and other drug use/misuse and other maladaptive coping strategies (e.g., aggression for SS youth). Youth were encouraged to discuss the short-term reinforcing properties of alcohol, as an attempt to help them understand their coping and conformity or enhancement motives for use, respectively. This was followed by a discussion of the long-term negative consequences of alcohol and other drug use/ misuse.

The coping skills portions of the group activity involved motivation-specific behavioural strategies and cognitive restructuring training taken from previous empirically-supported interventions with majority culture teens (Comeau 2004; Conrod et al. 2006). The coping skills training sections also included discussion of "scenarios" (i.e., stories from at-risk teens' real lives) that were informed by the qualitative thematic analyses of Study 2 (Stage 3) data in which context and consequences of risky or maladaptive behaviour (including but not limited to alcohol use) were generated. In addition to cognitive restructuring, the interventions also involved the use of exercises in which youth engaged in activities designed to induce automatic thoughts. Adolescents were simultaneously instructed by the facilitator to utilize cognitive restructuring techniques to counter such thoughts (Comeau 2004; Conrod et al. 2006).

The early interventions were developed in handbook form involving a manual for the facilitators and a workbook for the participants which is a subset of the material found in the facilitator manual (C2004 6148042 Canada Inc., Dr. Sherry H. Stewart, Dr. Patricia J. Conrod, Javin Creative Inc.). The manuals and workbooks made use of images that, like the scenarios, were informed by the results of quantitative Study 1 and qualitative Study 2 . The investigators worked with Mi'kmaq youth artists to capture the contextual and emotional content of the stories (Comeau et al. 2005a, b, c). For example, the three groups of teens' diverse experiences and contexts of alcohol use, based on the multiplicity of their social and cultural identities, interests, and experiences, were documented and conveyed in the introductory composite images. Several of the scenarios tried to capture the complexities of teens' social and personal relationships with alcohol as the youth in Study 2 defined these relations. Some scenarios focused on maladaptive coping strategies in an attempt to document the interviewed teens' diverse experiences with and contexts of alcohol use. The scenarios were designed to illustrate specific functions of alcohol and to illustrate adolescents' stories of how drinking for specific reasons (e.g., coping, conformity, enhancement) could lead to the situation "spiralling out of control". The manuals and interventions provided space for the students to creatively document and express their active choices, personal strengths, and priorities. The manuals also included Mi'kmaq 
language, holistic teachings, and images to illustrate concepts such as wholeness and balance.

\section{Stage 4}

\section{Method}

The Stage 4 outcome evaluation included focus group sessions and a pilot test. While it is an empirical question whether interventions need to be adapted for cultural relevance to be effective, we predicted our approach including the initial engagement of First Nation teens, would have meaning for these youth versus generic approaches not adapted for cultural relevance. Focus groups with participating students as well as facilitators and co-facilitators were conducted after the interventions were completed to get their perspectives on what was working and what needed changing about the interventions and manuals (Stevens and Comeau 2005).

\section{Results}

Focus group discussion highlighted the importance of taking into account community context, community history and participating school dynamics - all factors which can influence program delivery. It was recommended that the program be extended from $2 \times 90$ minute sessions to include $4 \times 45 \mathrm{~min}$ sessions to allow for flexibility of school schedules and retain student interest over time; the initial 90-minute format was experienced as too long for some students. It was also recommended that participating students have the option of continuing to meet as a group following program delivery to assist with ongoing student support. Monitoring treatment integrity and the length of service delivery of such ongoing student support sessions would be difficult to test in a controlled trial research design.

In addition to focus group evaluation, the efficacy of this set of interventions was tested in high schools in two rural First Nation communities in Nova Scotia. The results of this pilot study have been presented in detail elsewhere (Mushquash et al. 2007). Briefly, students were assigned to the appropriate personality group intervention (group format; 2 sessions) and then re-assessed 4 months later. The interventions were conducted in identical fashion at the two sites. An open trial was conducted which demonstrated that the interventions were associated with significant reductions in drinking frequency, frequency of binge drinking, drinking-related problems, and marijuana use, and significant increases in alcohol abstinence rates, relative to the normal developmental course of drinking/other substance use behaviour in a comparison group ${ }^{3}$ (Mushquash et al. 2007).

\section{Discussion}

The personality-based approach to the early intervention of alcohol problems in youth appears to be a promising method for reducing drinking behaviour and early signs of drinking problems not only for majority culture youth (e.g., Conrod et al. 2006) but also for

\footnotetext{
${ }^{3}$ The comparison group was comprised of student drinkers with AS, HOP, or SS personality characteristics, who had volunteered to participate in the intervention, but did not attend for a variety of reasons (e.g., absent from school during intervention).
} 
First Nations adolescents, at least when the approach is appropriately culturally adapted. In an open-trial pilot study, the brief interventions were shown to be effective in facilitating abstinence, reducing drinking quantity and binge drinking rates, reducing alcohol problems, and reducing marijuana use in First Nations youth relative to no intervention (Mushquash et al. 2007). These are important findings in light of the recognition that those who initiate drinking in early youth are more likely to increase their drinking, to experience alcoholrelated problems during adolescence, and are at greater risk for life-time alcohol abuse or alcoholism (Hawkins et al. 1997).

Assuming future controlled trials research establishes this set of interventions as effective, this four-stage methodology should optimally open avenues for school-based, substance abuse policy and procedure for innovative student assistance mechanisms while also strengthening partnerships among community stakeholders with youth as their mandate. Detailed discussion with respect to the importance of enhancing the collaborative relationships between researchers and community members, as well as culturally relevant approaches to assessment and measurement have been presented in more detail elsewhere (see Mushquash and Bova 2005; Mushquash et al. 2007, 2008).

Considering the elevated and problematic co-occurrence between addictive and nonaddictive disorders in youth (Conrod and Stewart 2005; Stewart and Conrod 2005; Zahradnik et al. 2007), this novel methodology and associated cognitive behavioural therapeutic intervention techniques present clinical advantages over other treatment strategies because this new approach has the possible advantage of improving coping skills related to both the substance abuse and co-morbid disorders (Comeau 2004; Conrod et al. 2006). Castellanos and Conrod (2006) showed that a U.K.-tailored version of the AS intervention reduced panic and school avoidance, the HOP intervention reduced depression, and the SS (i.e. impulsivity) intervention reduced acting-out behaviours such as shop lifting, for high-risk adolescents in inner city London.

This four-stage methodology is an important basic framework that can result in effective new interventions for First Nations youth by building community-researcher partnerships. These partnerships have the potential to allow for future projects that benefit the communities by meeting the priority needs of the communities as defined by them. However, an informal, but arguably most important feature needs to be emphasized: the strength and capability of youth to survive and thrive. Aboriginal traditions are a foundation from which we can not only survive but thrive as a people. Aboriginal youth are willing to share their gifts if we are willing to listen to and hear their voices. Guided by a strong heart we will build a good way to grow from the foundation of teachings shared to us from the Elders.

Acknowledgements Funding support for the methodology within First Nation communities was provided by the Canadian Institutes of Health Research (CIHR) New Emerging Team grant, a CIHR Investigator Award to Dr. Sherry Stewart, Dalhousie University, the Royal Canadian Mounted Police (RCMP) Foundation, and the Canada Research Chairs Program, Integrative Science, SSHRC. The authors of this paper wish to thank the First Nations adolescents who participated in this research; the Elders who provided their experience, knowledge, and expertise in guiding the early work; and the school personnel who were integral in all aspects of data collection. We also thank Pamela Collins, Ellen Rhyno, Marc Zahradnik and all members of Anxiety \& Alcohol Research Laboratory, Dalhousie University for their support. We gratefully acknowledge Dr. Cheryl Bartlett, Director Institute for Integrative Science and Health, Integrative science students and team members of Cape Breton University, Nova Scotia who contributed to this project by facilitating and financially supporting our attendance and inclusion in a cultural exchange on health and healing in a First Nations community in Nova Scotia. Special thanks are extended to Royal Canadian Mounted Police Inspector David Wojcik and participating members of the Aboriginal \& Diversity Policing Services "H" Division (Nova Scotia) for their significant financial and in-kind contribution, inspiration and leadership. We would also like to acknowledge the staff, counselors and students of participating schools and 
the Mi'kmaq First Nation communities. Finally, we would like to thank artist Vincent Walsh for communication design and Alexa Thompson for her production assistance in manual layout.

\section{References}

Aboriginal Healing Foundation. (2004). Historic trauma and aboriginal healing. Ottawa: Author.

Aboriginal Healing Foundation. (2005). Warrior-caregivers: Understanding the challenges and healing of First Nations men. A resource guide. Author: Ottawa.

Aboriginal Healing Foundation. (2006). Final report of the aboriginal healing foundation. Volume I. A healing journey: Reclaiming wellness. Ottawa: Author.

Abrams, D. B., \& Clayton, R. (2001). Transdisciplinary research to improve brief interventions for addictive behaviours. In P. M. Monti, S. M. Colby \& T. A. O'Leary (Eds.), Adolescents, alcohol and substance abuse: Reaching teens through brief interventions. New York: Guilford.

Ball, J. (2006). Early childhood care and development programs as hook and hub: Promising practices in First Nations communities. University of Victoria. School of Child and Youth Care, Early Childhood Development Intercultural Partnerships Programs Report. Victoria, British Columbia, Canada.

Brokenleg, M. (2002). The circle of courage. In L. K. Brendtro, M. Brokenleg \& S. V. Bockern (Eds.), Reclaiming youth at risk: Our hope for the future (pp. 43-68). Bloomington: National Educational Service.

Castellanos, N., \& Conrod, P. (2006). Brief interventions targeting personality risk factors for adolescent substance misuse reduce depression, panic, and risk-taking behaviours. Journal of Mental Health, 15, 645-658.

Chandler, M. J., Lalonde, C. E., Sokol, B., \& Hallett, D. (2003). Personal persistence, identity development, and suicide: a study of native and non-native north American adolescents. Monographs of the Society for Research in Child Development, 68(2), Serial No. 273.

Comeau, M. N. (2004). Framing solutions: Adolescent girls and their relationships with alcohol. (Doctoral Dissertation, Dalhousie University, 2004). Dissertation Abstracts International, 64, 4601B.

Comeau, N., \& Stewart, S. (2004). A qualitative study of self-perceived reasons for drinking in a sample of high personality risk First Nation adolescent drinkers: Toronto, ON, Canada, November, 2004.

Comeau, M. N., \& Stewart, S. H. (2005). A novel methodology for the development of adolescent alcohol abuse early interventions: Application to First Nations youth. Presented at a symposium on The nexus of health and culture I: Alcohol, youth, and interventions (Chair: Dr. Randal Tonks) at the Canadian Psychological Association Annual Convention, Montreal, PQ, Canada.

Comeau, N., Stewart, S. H., \& Loba, P. (2001). The relations of trait anxiety, anxiety sensitivity, and sensation seeking to adolescents' motivations for alcohol, cigarette, and marijuana use. Addictive Behaviours, 26, 803-825.

Comeau, M. N., Stewart, S. H., Loba, P., \& Theakston, J. (2004). A novel methodology for the development of adolescent alcohol abuse preventative interventions. Alcoholism: Clinical and Experimental Research, 28(Suppl. 5), 179A.

Comeau, M. N., Stevens, D., Mushquash, C., \& Stewart, S. H. (2005a). Indigenous knowledge guiding youth self-healing practices. Presented at the Canadian Aboriginal Science and Technology Society (CASTS) Conference: "Spirit of the East". Sydney, NS, September.

Comeau, M.N., Stewart, S.H., Mushquash, C., Wojcik, D., Bartlett, C., Marshall, M., Young, J., MacKenzie, J., Stevens, D., \& Collins, P. (2005b). Development and evaluation of a culturally-relevant alcohol abuse early intervention program for Mi'kmaq First Nation youth. Presented at the 6th Conference of the Canadian Rural Health Research Society (CRHRS) and the 1st Conference of the Canadian Society for Circumpolar Health, Quebec City, October.

Comeau, M. N., Stewart, S. H., Mushquash, C., Wojcik, D., Bartlett, C., Marshall, M., et al. (2005c). Community collaboration in developing a culturally-relevant alcohol abuse early intervention program for First Nation youth. Ontario Association of Children's Aid Societies Journal, 49, 39-46.

Conrod, P. J., \& Stewart, S. H. (2005). A critical look at dual-focused cognitive-behavioural treatments for comorbid substance use and psychiatric disorders: strengths, limitations, and future directions. Journal of Cognitive Psychotherapy, 19, 261-284.

Conrod, P. J., Pihl, R. O., \& Vassileva, J. (1998). Differential sensitivity to alcohol reinforcement in groups of men at risk for distinct alcoholism syndromes. Alcoholism: Clinical \& Experimental Research, 22, 585-597.

Conrod, P. J., Pihl, R. O., Stewart, S. H., \& Dongier, M. (2000a). Validation of a system of classifying female substance abusers based on personality and motivational risk factors for substance abuse. Psychology of Addictive Behaviours, 14, 243-256. 
Conrod, P. J., Stewart, S. H., Pihl, R. O., Côté, S., Fontaine, V., \& Dongier, M. (2000b). Efficacy of brief coping skills interventions that match different personality profiles of female substance abusers. Psychology of Addictive Behaviours, 14, 231-242.

Conrod, P. J., Stewart, S. H., Comeau, M. N., \& Maclean, M. (2006). Efficacy of cognitive behavioural interventions targeting personality risk factors for youth alcohol misuse. Journal of Clinical Child and Adolescent Psychology, 35, 550-563.

Conrod, P. J., Castellanos, N., \& Mackie, C. (2008). Personality-targeted interventions delay the growth of adolescent drinking and binge drinking. Journal of Child Psychology and Psychiatry, 49, 181-190.

Cooper, M. L. (1994). Motivations for alcohol use among adolescents: development and validation of a fourfactor model. Psychological Assessment, 6, 117-128.

Cooper, M. L., Frone, M. R., Russell, M., \& Mudar, P. (1995). Drinking to regulate positive and negative emotions: a motivational model of alcohol use. Journal of Personality and Social Psychology, 69, 990-1005.

Hawkins, J. D., Graham, J. W., Maguin, E., Abbott, R., Hill, K. G., \& Catalano, R. F. (1997). Exploring the effects of age on alcohol use initiation and psychosocial risk factors on subsequent alcohol misuse. Journal of Studies on Alcohol, 58, 280-290.

Kaminer, Y. (1999). Addictive disorders in adolescents. Addictive Disorders, 22, 275-287.

Kirmayer, L. J., Brass, G. M., Tait, C. L. (2001). The mental health of aboriginal peoples (pp. 5-25). Proceeding of the Advanced Study Institute McGill Summer Program in Social \& Cultural Psychiatry and the Aboriginal Mental Health Research Team. Montreal, Canada.

Marlatt, G. A., \& Miller, W. R. (1984). The comprehensive drinker profile. Odessa: Psychological Assessment Resources, Inc.

Ministries of Education Working Group: Alberta, British Columbia, Manitoba, Yukon Territory, Northwest Territories, Saskatchewan. (2000). The Common Curriculum Framework for Aboriginal Language and Cultural Programs: Kindergarten to Grade 12. Western Canadian Protocol for Collaboration in Basic Education. www.wcp.ca.

Mushquash, C. J., \& Bova, D. L. (2005). Cross-cultural assessment and measurement issues. Journal on Developmental Disabilities, 13, 53-66.

Mushquash, C., Comeau, N., \& Stewart, S. (2007). An alcohol abuse early intervention for First Nations adolescents. The First Peoples Child and Family Review, 3, 17-26.

Mushquash, C., Stewart, S., Comeau, M. N., \& McGrath, P. (2008). The structure of drinking motives in First Nations adolescents in Nova Scotia. American Indian and Alaska Native Mental Health Research: The Journal of the National Center, 15, 33-52.

Pihl, R. O., \& Peterson, J. B. (1995). Alcoholism: the role of different motivational systems. Journal of Psychiatry and Neuroscience, 20, 372-296.

Poulin, C., \& Wilbur, B. (2002). Nova Scotia student drug use 2002 highlights report. Halifax: Nova Scotia Department of Health.

Stevens, D., \& Comeau, M. N. (2005). Listening to youth: A qualitative evaluation of a first nation adolescent alcohol abuse preventative intervention program. Presented at the Canadian Centre on Substance Abuse (CCSA) 1st National Conference, Toronto, Canada, November.

Stewart, S. H., \& Conrod, P. J. (2005). Introduction to the special issue on state-of-the-art in cognitivebehavioural interventions for substance use disorders. Journal of Cognitive Psychotherapy, 19, 195-198.

Stewart, S. H., \& Devine, H. (2000). Relations between personality and drinking motives in young adults. Personality and Individual Differences, 29, 495-511.

Stewart, S. H., \& Zeitlin, S. B. (1995). Anxiety sensitivity and alcohol use motives. Journal of Anxiety Disorders, 9, 229-240.

Stewart, S. H., Karp, J., Pihl, R. O., \& Peterson, R. A. (1997). Anxiety sensitivity and self-reported reasons for drug use. Journal of Substance Abuse, 9, 223-240.

Stewart, S. H., Loughlin, H. L., \& Rhyno, E. (2001). An examination of the mediating roles of internal drinking motives in explaining personality domain-drinking behaviour relations in young adults. Personality and Individual Differences, 30, 271-286.

Stewart, S. H., Comeau, M. N., Mushquash, C., Bartlett, C., Marshall, M., Young, J., et al. (2005a). Community collaboration in the development \& evaluation of a culturally-relevant alcohol abuse early intervention program for First Nation youth. Presented at a symposium on "New Developments in Early Intervention for Alcohol Abuse among First Nations Youth" (Co-Chairs: Drs M.N. Comeau and S.H. Stewart) at the Canadian Centre on Substance Abuse (CCSA) 1st National Conference, Toronto, ON, November 13-16.

Stewart, S. H., Conrod, P. J., Marlatt, G. M., Comeau, M. N., Thush, C., \& Krank, M. (2005b). New developments in prevention and early intervention for alcohol abuse in youth. Alcoholism: Clinical and Experimental Research, 29, 278-286. 
Stewart, S. H., English, K., \& Comeau, N. (2005c). Relations between personality variables and risky drinking motives in aboriginal adolescents. Presented at the $31 \mathrm{st}$ Annual Dalhousie University Department of Psychology In-House Convention, Halifax, NS, April.

Stewart, S. H., English, K., \& Comeau, M. N. (2005d). Relations between personality variables and risky drinking motives in Aboriginal adolescents. Presented at the 27th Annual Scientific Meeting of the Research Society on Alcoholism (RSA), Santa Barbara, CA.

Theakston, J. A., Stewart, S. H., Dawson, M., Knowlden, S. A. B., \& Lehman, D. R. (2004). Big five personality domains predict drinking motives. Personality and Individual Differences, 37, 971-984.

Zahradnik, M., Stevens, D. S., Stewart, S. H., Comeau, N. M., Wekerle, C., \& Mushquash, C. (2007). Building a collaborative understanding of pathways to adolescent alcohol misuse in a Mi'kmaq community: a process paper. The First Peoples Child and Family Review, 3, 27-36. 